\title{
Las contribuciones del espacio costero marino para pueblos originarios (ECMPO) al bienestar humano de las comunidades indígenas de Carelmapu, Sur de Chile ${ }^{1}$
}

Contributions of indigenous marine areas to human well-being of indigenous people of CARELMAPU, southern Chile

\author{
Daniça Cid \\ Francisco Araos \\ Universidad de Los Lagos, Chile
}

RESUMEN Los Espacios Costeros Marinos para Pueblos Originarios (ECMPOs) se han transformado en el principal mecanismo para el resguardo de los modos de vida de los pueblos indígenas y promoción de la sustentabilidad marinocostera del sur de Chile. El artículo analiza las contribuciones de los ECMPOs al bienestar humano de las comunidades indígenas. A través de una investigación de carácter cualitativo, desarrollada en la localidad de Carelmapu, Comuna de Maullín, Región de los Lagos, se presentan potenciales indicadores de bienestar humano asociados a la creación e implementación del ECMPO. El análisis demuestra que la conservación de la biodiversidad y el resguardo de la calidad ambiental de los ecosistemas marino-costeros promovidos por los ECMPOs son componentes esenciales para el bienestar humano, cuya degradación influiría en la capacidad de los ecosistemas de generar los servicios ambientales que sustentan la vida en la localidad.

PALABRAS CLAVE Bienestar humano; espacios costeros marinos para pueblos originarios; comunidades indígenas; sustentabilidad.

1. Agradecimiento a Proyecto ANID/FONDECYT N. 11180066 "ECMPOs: Ensamblajes conservacionistas para la protección de lugares de vida en la zona costera de la Región de Los Lagos". A las Comunidades Indígenas de Carelmapu. 


\begin{abstract}
Indigenous Marine Areas (ECMPO in Spanish) are the main institutional arrangement for the protection of the indigenous people's livelihoods and the promotion of marine sustainability of southern Chile. This article analyzes the contributions of ECMPO to the human well-being of indigenous communities. Through qualitative research conducted in the coastal town of. Carelmapu, Municipality of Maullín, Region de Los Lagos, we present potential human well-being indicators associated with the creation and implementation of ECMPO. The analysis shows that biodiversity conservation and the environmental quality of marine-coastal ecosystems promoted by ECMPO are essential components for the human well-being of indigenous communities, whose degradation might influence the capacity of the ecosystems to provide environmental services that sustain the life in the locality.
\end{abstract}

KEYWORDS Human well-being; indigenous marine areas; indicators; sustainability.

\title{
Introducción
}

El bienestar humano es un concepto multidisciplinar que a lo largo de la historia ha sido considerado un objetivo común y universal de la humanidad (Aguado et al., 2012). Se ha definido el bienestar humano como el grado en que las necesidades de las personas se satisfacen en relación a percepciones subjetivas de carácter individual o colectivo (Costanza et al., 2007). El análisis del bienestar humano se ha basado, principalmente, en dos grandes aproximaciones: la primera fundamentada en aspectos psicológicos y subjetivos, que postula que el bienestar de una persona radica en la realización y protección del "ser", sin importar el contexto en el que se inscribe su existencia; mientras que por otro lado, un enfoque económico que apuesta por una conexión del bienestar humano con el nivel de ingresos y la satisfacción de necesidades materiales, actualmente el más usado a nivel internacional y que, por ejemplo, se mide a través Producto Interno Bruto (PIB) per cápita (Aguado, 2016).

El ser humano está en constante interacción con su medio natural, que le provee de los soportes esenciales para su vida, ya sea para su abastecimiento, recreación y el mantenimiento de sus prácticas culturales (Costanza et al., 2019). Estos aportes de los sistemas naturales a los seres humanos han sido denominados servicios ambientales o ecosistémicos, entendiéndolos como el conjunto de beneficios que los ecosistemas entregan a la humanidad, tales como: servicios de provisión (peces, bosques, pastos), servicios de regulación (polinización), soporte (producción de oxígeno, suelos fértiles), culturales (identidad, sentido de pertenencia) (MEA, 2005). 
De esta manera, en la actualidad se reconoce una estrecha relación de los servicios ecosistémicos con el bienestar humano, siendo fundamental el mantenimiento de la biodiversidad que albergan los territorios habitados por pueblos indígenas y comunidades locales (Pascual et al., 2017). Actualmente, los medios de vida de más de tres millones de personas en el mundo dependen de la biodiversidad marino-costera, por lo que se plantea a nivel mundial y por medio de los Objetivos de Desarrollo Sostenible (ODS) la necesidad de incentivar la conservación y el uso sostenible de los recursos marinos (Objetivo 14). Para la plataforma científica internacional Millennium Ecosystem Assessment (MEA) el bienestar humano considera cinco dominios principales: Seguridad, Materiales Básicos para una Buena Vida, Salud, Buenas relaciones sociales y Libertad de Elección y Acción (MEA, 2005). En cada caso, los dominios identificados son representados a través de un conjunto de atributos e indicadores particulares.

Se considera que el análisis del bienestar humano asociado a la protección de sistemas naturales tiene gran potencial, tanto en el reconocimiento de la dimensión humana en la gestión de la biodiversidad, como para el desarrollo de métricas e indicadores que evalúen de manera concreta el alcance e impactos de la conservación en las distintas dimensiones de la vida de las comunidades asociadas a las áreas protegidas (Kilpatrick, Salkeld, Titcomb y Hahn, 2017).

Desde una perspectiva intercultural, en la cosmovisión mapuche el Kume Mongen (buen vivir) se concibe como el anhelo de la vida en armonía con todos los seres (Rojas y Soto, 2016), donde la relación del ser humano con su medio natural y su entorno social se vuelve fundamental para alcanzar el equilibrio espiritual.

El Kume Mongen representa una inagotable fuente de saberes, siendo el horizonte desde donde se crea el modo de vida del pueblo mapuche, pues es el anhelo de una vida en armonía que abarca desde el estar en equilibrio con uno mismo, con la comunidad y el entorno natural. La enfermedad, por lo tanto, surge como manifestación de los desequilibrios socio-ecológicos y comunitarios, cuya sanación requiere del restablecimiento del orden social y natural (Hasen, 2012).

De acuerdo con lo planteado, se identifica una sinergia entre el bienestar humano basado en los servicios ecosistémicos, el buen vivir mapuche y la implementación de la ley Lafkenche (Ley 20.249/2008), por medio de la creación de Espacios Costeros Marinos para Pueblos Originarios (ECMPOs). Consideramos que el resguardo de los usos consuetudinarios de los pueblos indígenas y la gestión local de los recursos naturales propiciadas por los ECMPOs, incentivan el cuidado de la biodiversidad, el manejo sustentable de los ecosistemas, la restauración de áreas degradadas ambientalmente $y$, en consecuencia, nutren una nueva forma de habitar el territorio/maritorio bajo una relación de convivencia entre seres humanos y la naturaleza (Araos et al., 2020b). 
De esta manera, el artículo busca desarrollar potenciales indicadores de las contribuciones de los ECMPO al bienestar humano de las comunidades indígenas, mediante un estudio de caso en la localidad de Carelmapu, al sur de Chile, donde actualmente seis comunidades indígenas lideran la solicitud del "ECMPO Carelmapu" desde el 2018.

Tabla 1. Teorías e Indicadores de Bienestar Humano.

\begin{tabular}{|c|c|c|c|c|}
\hline \multicolumn{5}{|c|}{ INDICADORES DE BIENESTAR } \\
\hline Teoría Integral & Kume Mongen & $\begin{array}{c}\text { Bienestar } \\
\text { Psicológico }\end{array}$ & $\begin{array}{l}\text { Paradoja } \\
\text { ingreso- } \\
\text { felicidad }\end{array}$ & $\begin{array}{l}\text { Millenium } \\
\text { Ecosystem } \\
\text { Assessment } \\
\text { (MEA) }\end{array}$ \\
\hline Justicia Social & $\begin{array}{c}\text { Relación con el } \\
\text { entorno social }\end{array}$ & $\begin{array}{c}\text { Satisfacción con } \\
\text { la vida }\end{array}$ & P.I.B & Biodiversidad \\
\hline $\begin{array}{l}\text { Relaciones so- } \\
\text { ciales y con la } \\
\text { naturaleza }\end{array}$ & $\begin{array}{c}\text { Relación con el } \\
\text { medio natural }\end{array}$ & de $\begin{array}{c}\text { Respuesta } \\
\text { relaciones } \\
\text { afectivas }\end{array}$ & $\begin{array}{l}\text { Nivel de } \\
\text { ingresos }\end{array}$ & $\begin{array}{c}\text { Mantención } \\
\text { de recursos } \\
\text { naturales }\end{array}$ \\
\hline Salud & $\begin{array}{c}\text { Equilibrio espir- } \\
\text { itual }\end{array}$ & Salud mental & $\begin{array}{c}\text { Tasa de } \\
\text { desempleo }\end{array}$ & Alteración \\
\hline Seguridad & Prácticas cul- & Felicidad & Consumo & de los ecosiste- \\
\hline Igualdad & turales & & $\begin{array}{c}\text { de bienes y } \\
\text { servicios }\end{array}$ & \\
\hline
\end{tabular}

Fuente: elaboración propia en base a Aguado et al. (2012), Hasen (2012),García et al. (2000), Eartland (2010), Millenium Ecosystem Assessment (2005).

\section{Ley Lafkenche y espacios costeros marinos para pueblos originarios}

La ley 20.249 más conocida como Ley Lafkenche se promulga en el año 2008, permitiendo la creación de los Espacios Costeros Marinos para Pueblos Originarios. Esta ley surgió producto de la movilización política de las comunidades indígenas asentadas en las regiones del sur del país, quienes buscaron el reconocimiento legal de las demandas territoriales sobre el borde costero a través del resguardo de sus usos consuetudinarios (Araos et al., 2020a; Gissi, Ibacache, Pardo y Ñancucheo, 2017; Nahuelpan, 2016).

Los ECMPOs, operan institucionalmente como una destinación o concesión marítima, cuya administración es entregada a una comunidad o asociación de comunidades indígenas legalmente reconocidas, previa aprobación del Plan de Administración del ECMPO por parte de una Comisión Intersectorial.

Esta figura legal tiene el objetivo de proteger el uso consuetudinario de los pueblos indígenas, los cuales de acuerdo al artículo $6^{\circ}$ inciso segundo de la Ley 20.249 se define como: "las prácticas o conductas realizadas por la generalidad de los integrantes de la comunidad o asociación de comunidades según corresponda, de manera habitual y 
que sean reconocidas colectivamente como manifestaciones de su cultura". Los usos consuetudinarios pueden comprender, entre otros, el uso pesquero, religioso, recreativo y/o medicinal.

Los ECMPOs pueden incluir zonas del borde costero que se encuentran bajo la administración del Ministerio de Defensa Nacional incluyendo: playas de mar, terrenos de playa fiscales, porciones de agua y fondo, y rocas, dentro y fuera de las bahías, entre otros. La superficie de los ECMPOs se determina de acuerdo al espacio geográfico necesario para asegurar el ejercicio de los usos consuetudinarios reconocidos por la ley. No obstante, los ECMPOs deben reconocer derechos de uso entregados con anterioridad: concesiones Marítimas y Áreas de Manejo y Explotación de Recursos Bentónicos, otorgadas a titulares distintos de las comunidades indígenas solicitantes. Desde su promulgación el año 2008 y hasta la actualidad, existen más de 96 solicitudes en trámite en el país, de las cuales solo 12 han sido decretadas, 11 de ellas en la Región de Los Lagos (Araos et al., 2020a). La lentitud del proceso se debe al amplio recorrido de la solicitud por distintos estamentos gubernamentales (SUBPESCA ${ }^{2}$, $\mathrm{CONADI}^{3}, \mathrm{CRUBC}^{4}$, entre otros), revelando las trabas institucionales y falta de claridad respecto a los criterios de aprobación que han caracterizado la implementación de la ley (Hiriart-Bertrand, Silva y Gelcich, 2020).

\section{Enfoque metodológico}

La investigación fue de carácter cualitativo (Canales, 2006), basada en el estudio de las prácticas y percepciones de las comunidades indígenas respecto de la situación actual y las proyecciones acerca del futuro del ECMPO Carelmapu.

La unidad de análisis fueron las comunidades indígenas de la localidad de Carelmapu, a través de sus dirigentes y líderes, quienes presentan un rango etario de 27 a 64 años. La asociación de comunidades indígenas solicitantes del ECMPO Carelmapu se compone de 06 comunidades: Comunidad Indígena Encuramapu, Comunidad Indígena Lafkenche Kupal, Comunidad Indígena Wetripantu, Comunidad Indígena Lafken Mapu, Comunidad Indígena Kalfu Lafken y Comunidad Indígena Huerque Mapu Lafken, reuniendo aproximadamente 150 socios.

El proceso de recopilación de información se conformó por dos etapas: i) la primera etapa incluyó el registro documental de documentos producidos por organismos públicos (Municipio, CONADI, Universidad de Los Lagos) y de ONGs (ONG Seacology); ii) la segunda etapa consistió en la realización de entrevistas en profundidad. En las entrevistas se abordaron temas como: la identificación de los beneficios

\footnotetext{
2. Subsecretaría de Pesca.

3. Corporación Nacional de Desarrollo Indígena.

4. Comisión Regional de Uso del Borde Costero.
} 
que entregan los espacios costeros marinos al bienestar de las comunidades indígenas en sus dimensiones ambiental, social, económica y cultural. Estas entrevistas fueron aplicadas a un informante clave de cada una de las comunidades indígenas. Para identificar a los entrevistados se utilizó la técnica de bola de nieve (Raworth, Sweetman, Narayan, Rowlands y Hopkins, 2012). De esta manera, se reconocieron los principales problemas, preocupaciones y anhelos relacionados con el ámbito territorial, dando cuenta de los beneficios que el ECMPO entrega actualmente y que aportaría en el futuro a la calidad de vida y bienestar en el territorio.

Con esta información, se procedió a la sistematización y análisis de los instrumentos por medio de categorías significativas operacionalizadas a través de una matriz de clasificación de la información en el software Atlas ti, herramienta que permitió segmentar los datos, codificarlos y establecer una visión relacional entre las categorías de análisis (Ver Tabla 2).

El trabajo de campo de la investigación se realizó entre Octubre de 2019 y Enero de 2020 , periodo en el cual se registraron cinco visitas a la localidad de Carelmapu. Finalmente, la información fue triangulada con los datos y análisis producidos por el Proyecto ANID/FONDECYT $11180066 \mathrm{del}$ cual los co-autores forman parte.

Tabla 2. Síntesis de la propuesta metodológica. Fuente: Elaborado por los autores.

\begin{tabular}{|c|c|c|c|c|}
\hline Metodología & $\begin{array}{c}\text { Unidad } \\
\text { de análisis }\end{array}$ & $\begin{array}{l}\text { Técnicas } \\
\text { utilizadas }\end{array}$ & $\begin{array}{c}\text { Tópicos de la } \\
\text { indagación }\end{array}$ & $\begin{array}{l}\text { Categorías } \\
\text { de análisis }\end{array}$ \\
\hline \multirow[t]{4}{*}{ Cualitativa } & \multirow{4}{*}{\begin{tabular}{|c|}
\multicolumn{2}{|c|}{06} \\
Comunidades \\
Indígenas de \\
la localidad de \\
Carelmapu, \\
Comuna de \\
Maullín, Región \\
de Los Lagos.
\end{tabular}} & \multirow{4}{*}{$\begin{array}{l}\text { - Registro } \\
\text { documental } \\
\text { información } \\
\text { pública de } \\
\text { ONGs } \\
\text { - Entrevista en } \\
\text { profundidad } \\
\text { a dirigentes } \\
\text { de comunidades } \\
\text { indígenas }\end{array}$} & $\begin{array}{c}\text { Contribuciones } \\
\text { Sociales de los } \\
\text { ECMPOs }\end{array}$ & $\begin{array}{c}\text { Participación } \\
\text { social } \\
\text { Desarrollo local } \\
\text { E m p o d er - } \\
\text { amiento }\end{array}$ \\
\hline & & & $\begin{array}{l}\text { Contribuciones } \\
\text { Económicas de } \\
\text { los ECMPOs }\end{array}$ & $\begin{array}{l}\text { Uso y manejo de } \\
\text { recursos } \\
\text { naturales }\end{array}$ \\
\hline & & & $\begin{array}{c}\text { Contribuciones } \\
\text { Ambientales de } \\
\text { los ECMPOs }\end{array}$ & $\begin{array}{c}\text { Biodiversidad } \\
\text { Amenazas } \\
\text { ambientales }\end{array}$ \\
\hline & & & $\begin{array}{l}\text { Contribuciones } \\
\text { Culturales de } \\
\text { los ECMPOs }\end{array}$ & $\begin{array}{l}\text { Buen vivir } \\
\text { Identidad } \\
\text { Prácticas } \\
\text { culturales }\end{array}$ \\
\hline
\end{tabular}




\section{Estudio de caso: Carelmapu, Tierra Verde}

El litoral chileno se caracteriza por presentar una costa continua y extensa, hasta su quiebre en el Canal de Chacao, donde comienzan archipiélagos, canales y fiordos. En el margen occidental del Canal de Chacao se encuentra la localidad costera de Carelmapu o "tierra verde" en Mapudungún (Cursach, 2018). Carelmapu es una localidad que cuenta con una superficie de 178,3 kilómetros cuadrados, donde habitan 3.537 habitantes (Instituto Nacional de Estadística, 2019). Gran parte de su actividad económica gira en torno a la extracción de recursos marinos, donde los principales usos son la explotación de recursos bentónicos ${ }^{5}$, la pesca en embarcación o de orilla, marisqueo o marisca, realizada en su mayoría por mujeres en las playas, y por último, la extracción de algas para el consumo y la comercialización (Rodríguez, Gajardo y Ther, 2014). Además de contar con una amplia diversidad marina, la localidad cuenta con lugares de relevancia ecológica, tales como sitios de reproducción de aves y mamíferos marinos, y un humedal costero ubicado en Lenqui considerado de importancia internacional para la conservación de aves playeras migratorias (Cursach, 2018).

La primera comunidad indígena organizada en el territorio fue la comunidad Encuramapu el año 2005. A partir del trabajo de socialización y reconocimiento indígena de sus miembros, se crean en los años siguientes otras comunidades indígenas: Kalfu Lafken, Lafkenche Kupal, Lafken Mapu. Finalmente, en el año 2018 se crean las últimas dos comunidades: Wetripantu y Huerque Mapu Lafken. En conjunto las comunidades conforman la Asociación de Comunidades Indígenas de Carelmapu, dando un total aproximado de 150 socios, quienes el año 2018 deciden solicitar un Espacio Costero Marino para Pueblos Originarios en su territorio. Sus dirigentes y líderes participan en distintas agrupaciones ligadas a la organización territorial, siendo agentes que están en constante interacción con otros actores ligados a la extracción de productos del mar.

5. Los recursos bentónicos son aquellas especies que se encuentran en las rocas o fondo marino, denominado bentos. Entre los recursos extraídos destacan: loco, erizo, pulpo, almeja, navajuela, picoroco, piure, culengue, jaibas, cangrejos. 


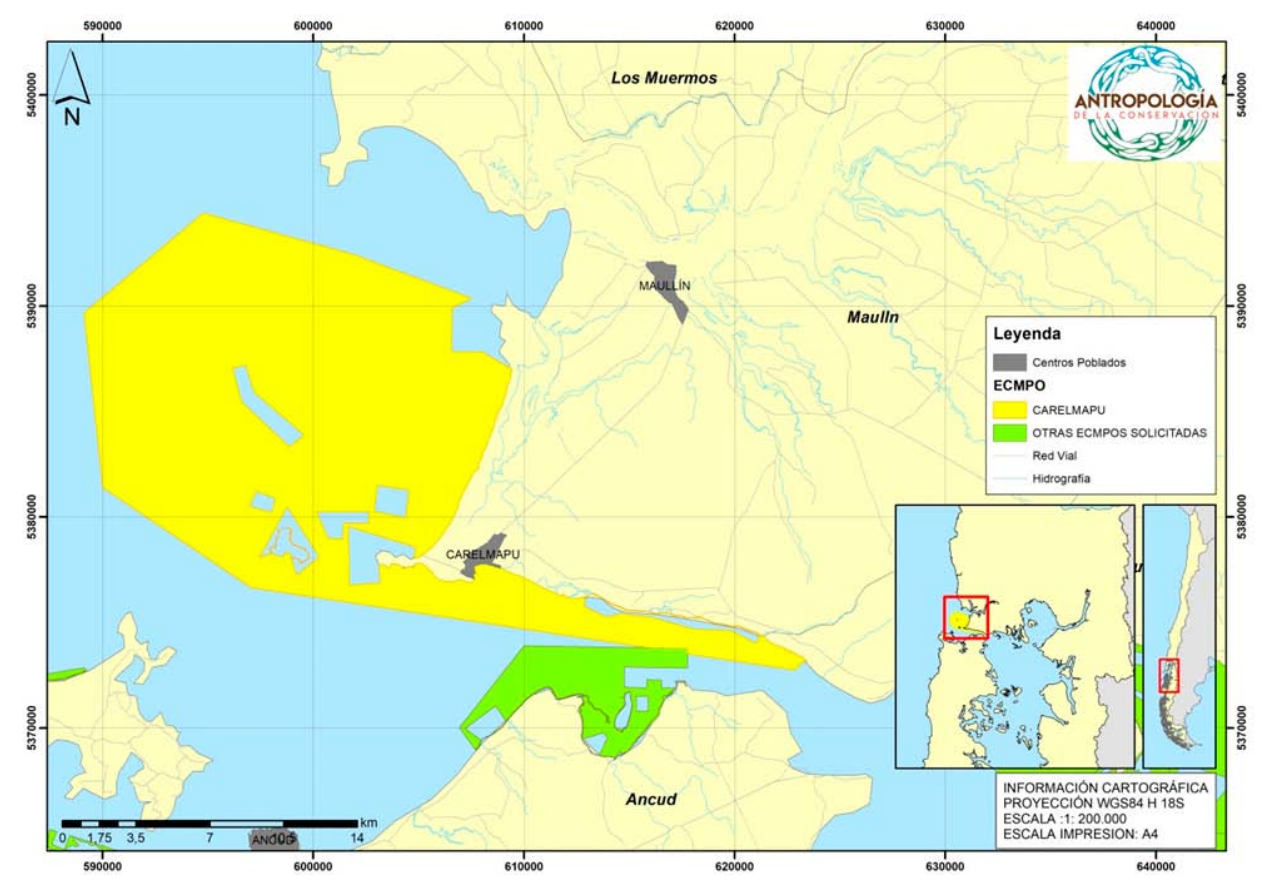

Figura 1. Mapa del Espacio Costero Marino para Pueblos Originarios de Carelmapu. Fuente: Elaborado por Francisco Brañas. Proyecto ANID/FONDECYT N. 11180066.

Para las comunidades indígenas el ECMPO emerge como una importante herramienta para la protección marino-costera de Carelmapu, tanto de amenazas de contaminación ambiental asociadas a la salmonicultura, como de la sobreexplotación local de los recursos naturales. La superficie del ECMPO solicitada alcanza las 28.106 hectáreas, abarcando desde el sector de Astilleros hasta Amortajado, pasando por la costa de la bahía de Carelmapu, Punta Chocoy e integrando a la Isla Doña Sebastiana y los Farellones de Carelmapu.

En cuanto al uso consuetudinario del espacio costero y marino, se destaca el uso pesquero, reconociéndose como la principal actividad económica de las comunidades indígenas. Entre estos usos se destacan: la pesca en embarcación, la marisca o marisqueo para el consumo doméstico y la recolección de algas. Asimismo, se identifica el uso religioso y/o espiritual por parte de las comunidades indígenas, reconociendo sitios ceremoniales en el sector de Los corrales y la Playa Mar Brava. Otro uso consuetudinario identificado es la recolección de lawen o hierbas medicinales, entre las que destacan el Sargazo o Huiro, la limpia plata, el agua de mar usada para dolores reumáticos y el aceite de lobo. En cuanto al uso recreativo, las comunidades identifican las playas de arena con las que cuenta el territorio como las principales zonas de esparcimiento para la comunidad, estas son: la Bahía de Carelmapu, Lenqui y Mar Brava. 
La importancia de la isla Doña Sebastiana para el territorio de Carelmapu radica en la conexión de las familias que realizan o realizaron en el pasado la extracción de productos marinos en el lugar. Los comuneros relatan historias y mitos sobre un barco hundido en la zona, así como de avistamientos del Caleuche ${ }^{6}$ y sirenas, que hacen de la isla Doña Sebastiana una parte importante del paisaje cultural de los habitantes de Carelmapu.

Las comunidades indígenas de Carelmapu reconocen la importancia que en la actualidad tiene la conservación de la naturaleza presente en el territorio, deseando que los usos consuetudinarios se orienten hacia la sustentabilidad y protección de los modos de la vida de las comunidades indígenas y locales.

\section{Resultados: ECMPOs y bienestar humano}

Al hablar de comunidades indígenas y su vínculo con el medioambiente, la protección de la biodiversidad y el manejo sustentable de los recursos naturales se torna relevante para la mantención de las prácticas comunitarias que sustentan sus modos de vida y su relación con los antepasados y ancestros. A partir del reconocimiento de estas prácticas, a continuación, se identifican, describen y analizan las potenciales contribuciones y beneficios de los ECMPOs al bienestar de los pueblos indígenas por medio del estudio de caso del ECMPO Carelmapu, en torno a cuatro grandes categorías: ambiental, social, económica y cultural.

En primera instancia se visualizan aportes del ECMPO en la dimensión ambiental, siendo fundamental para las comunidades otorgar una protección jurídica al territorio. Tal y como señala el presidente de una de las comunidades indígenas:

"Conscientes de la riqueza que tiene este lugar y del apego que tenemos hacia él y de la identidad que tenemos como territorio, yo creo que es fundamental otorgarle a este espacio un marco de protección jurídica que nos permita protegerlo"?

La protección jurídica del espacio se hace necesaria a partir de las constantes amenazas que enfrenta el territorio de Carelmapu por parte de grandes emprendimientos industriales que cada cierto tiempo se acercan e intentan instalarse en la zona. A propósito una dirigenta señala que "cuando se ingresó (el ECMPO) nos encontramos con la sorpresa de que aquí en Carelmapu había muchas empresas que querían venir"s.

\footnotetext{
6. Historia mitológica de la isla de Chiloé, la cual relata el avistamiento de un barco fantasma que deambula en la zona sur de Chile.

7. Entrevista realizada el 11 de Octubre de 2019. Carelmapu, Comuna de Maullín.

8. Entrevista realizada el 19 de Enero de 2020. Carelmapu, Comuna de Maullín.
} 
Existe además, interés por parte de las comunidades indígenas de preservar los recursos naturales que alberga el mar de Carelmapu: "lo que buscamos es proteger, no trabajar lo que está en veda, las especies fuera de medida, la idea es regularlo todo para que después tengamos"9.

Lo anterior cobra importancia cuando analizamos los cambios en la biodiversidad y la manera en que estas transformaciones impactan en el bienestar y calidad de vida de las personas, comprometiendo el funcionamiento del ecosistema y su capacidad de generar servicios para la sociedad (Martín-López et al., 2007). Actualmente, existen además malas prácticas en la localidad asociadas a la extracción de recursos marinos, ya sea por parte de los mismos pescadores o recolectores, como por turistas que no siguen protocolos o sistemas de manejo de los recursos. Como señala el dirigente de una de las comunidades:

"se supone que el recolector de orilla tiene que esperar que la luga se suelte de la piedra, se salga por sí sola, no que la vayas a arrancar"10.

Cabe destacar, que los miembros de las comunidades indígenas de Carelmapu realizan, en su mayoría, actividades laborales ligadas al mar, tales como: buzo mariscador, recolectores de orilla, desconchadoras de piure, entre otros, contando con experiencia y conocimientos tradicionales respecto a las formas de extracción de los recursos del mar.

En segunda instancia, se visualizan amplios aportes en la dimensión social. Referimos a un proyecto que requiere de un constante diálogo intra y extra comunitario durante el proceso de toma de decisiones, involucrando a todos los actores locales y usuarios de los recursos naturales de la zona. Al respecto, uno de los dirigentes menciona:

“acá tenemos la posibilidad de hacer las cosas distintas, de que nos pongamos de acuerdo y seamos nosotros quienes decidamos como queremos vivir, que cosas queremos que existan en este pueblo y que nos permita proyectar este pueblo al futuro"11.

De acuerdo a lo anterior, la participación ciudadana en la solicitud del ECMPO se vuelve un eje fundamental para el desarrollo local, contribuyendo a la descentralización de la toma de decisiones, ya que son los actores locales los llamados a decidir qué tipo de desarrollo es el más adecuado para su territorio (Delgado, Bachmann y Oñate, 2007). Otro de los ejes fundamentales que se identifican en materia social es la cali-

9. Entrevista realizada el 16 de Enero de 2020. Carelmapu, Comuna de Maullín.

10. Entrevista realizada el 19 de Enero de 2020. Carelmapu, Comuna de Maullín.

11. Entrevista realizada el 11 de Octubre de 2019. Carelmapu, Comuna de Maullín. 
dad de vida de los habitantes de la localidad, quienes a través de la solicitud visualizan una oportunidad para la mantención de sus modos de vida. A propósito, la presidenta de una de las comunidades indígenas señala:

"aquí aún hay olor a mar, es impagable el olor a mar $\{. .$.$\} yo defino así mi$ forma de vida, valorizo el estar acá y por eso es que estamos dando la pelea de que las empresas no se instalen en nuestro hogar" ${ }^{{ }^{12}}$.

En tercera instancia, se hace referencia a la dimensión económica. La base del desarrollo sostenible implica la comprensión de la relación entre seres humanos y naturaleza, derivada de la actual crisis ambiental que vive el planeta, proponiendo de esta manera una forma distinta de desarrollo local. De acuerdo a lo anterior, la narrativa de uno de los dirigentes es relevante para comprender las estrategias de las comunidades locales: "apostamos a una serie de emprendimientos, que no sean masivos y que sean sustentables, sostenibles y que sean desarrollados por las propias personas"13. Así también, coincide otra dirigenta: "me imagino mucha afluencia de turismo, con un pueblo mucho más... con hartos proyectos, pero proyectos sanos" ${ }^{14}$.

De esta forma, se plantea desde las comunidades indígenas un desarrollo económico para la localidad que integra formas y niveles de extracción de productos marinos que sean armónicos con el medio ambiente, garantizando la sostenibilidad de las actividades productivas y, a su vez, la posibilidad de una nueva fuente laboral.

Por último, se añade la dimensión cultural, donde las contribuciones refieren a la integración y fomento de manifestaciones culturales en el territorio, poniendo sobre la mesa una alternativa de desarrollo guiada por la cosmovisión mapuche. De acuerdo a lo anterior, un dirigente menciona que: "desde la cosmovisión mapuche hemos aprendido que somos una cadena, no hay nadie que esté sobre, por lo tanto de esta visión, tenemos la obligación de cuidar a todos los demás, si no se produce un desequilibrio"15.

De acuerdo a esto, se torna innegable la importancia del medio ambiente en la constitución cultural de los pueblos indígenas, ya que es un medio que no solo los dota de recursos, sino que también de identidad (Gaona, 2013). Para Hasen (2012) frente a la urgente necesidad de construir una alternativa de desarrollo para el planeta, es que nacen las opciones de miradas locales y de los pueblos originarios. No se trata de volver al pasado, ni negar los avances tecnológicos actuales, sino que de incorporar a nuestras miradas e ideas otra forma de entender el mundo que nos rodea,

12. Entrevista realizada el 19 de Enero de 2020. Carelmapu, Comuna de Maullín.

13. Entrevista realizada el 11 de Octubre de 2019. Carelmapu, Comuna de Maullín.

14. Entrevista realizada el 22 de Enero de 2020. Carelmapu, Comuna de Maullín.

15. Entrevista realizada el 11 de Octubre de 2020. Carelmapu, Comuna de Maullín. 
una ética ambiental convocante e inclusiva (Araos et al., 202ob). Tal y como señala un dirigente: "no indica que el día de mañana todos sean mapuche, sino que la comprendan y la hagan suya, como es los elementos en relación con el medio ambiente"16.

\section{Indicadores de bienestar humano del ECMPO Carelmapu}

Con el objetivo de operacionalizar las dimensiones del bienestar humano exploradas, se presentan a continuación potenciales indicadores que reflejan las contribuciones y beneficios del ECMPO al bienestar humano de la comunidad de Carelmapu, haciendo una breve descripción de cada uno de ellos. Cabe destacar que los indicadores se presentan como una propuesta de variables sintéticas que permiten contextualizar y situar el bienestar humano en un territorio en particular.

\begin{tabular}{|c|c|c|c|c|c|c|c|c|c|c|c|c|c|c|}
\hline \multirow{2}{*}{ Comunidades Indigenas de Carelmapu } & \multicolumn{6}{|c|}{ DEMENSIÓN SOCIAL } & \multicolumn{2}{|c|}{$\begin{array}{l}\text { DIMIENSIÓN } \\
\text { AMIBIENTAL }\end{array}$} & \multicolumn{2}{|c|}{$\begin{array}{l}\text { DINENSIÓN } \\
\text { ECONÓMICA }\end{array}$} & \multicolumn{4}{|c|}{ DINENSIÓN CULTURAL } \\
\hline & P.C & E.A & D.L & E & c.v & D.P & P.B & v.A & S.R & D.L & B.V & A.L.D & F.M.C & I.T \\
\hline Comumidad Indigena Encura Mapu. & & & & & & & & & & & & & & \\
\hline Comumidad Indigena Lafkenche Kupal & & & & & & & & & & & & & & \\
\hline Comunidad Indigena Lafken Mapu & & & & & & & & & & & & & & \\
\hline Comunidad Indigena Kalfu Lafken & & & & & & & & & & & & & & \\
\hline Comumidad Indigena Wetripantu & & & & & & & & & & & & & & \\
\hline Comumidad Indigena Huerque Mapu Lafken & & & & & & & & & & & & & & \\
\hline
\end{tabular}

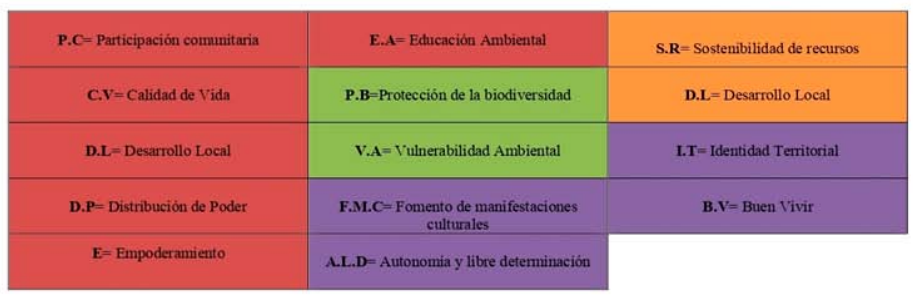

Figura 2. Potenciales Indicadores del bienestar humano del ECMPO a las comunidades indígenas de Carelmapu.

\section{Contribuciones ambientales}

Protección de la biodiversidad: La biodiversidad o diversidad biológica, definida como la variedad de formas de vida en la Tierra, está actualmente en una trayectoria de disminución, afectando la capacidad del medio ambiente de contribuir al bienestar de las personas (IPBES, 2020) ${ }^{17}$. Carelmapu se caracteriza por acoger una amplia

16. Entrevista realizada el 11 de Octubre de 2020. Carelmapu, Comuna de Maullín.

17. Plataforma Intergubernamental sobre Diversidad Biológica y Servicios de los Ecosistemas, IPBES. Consultado el 19 de Enero de 2020. 
biodiversidad, destacándose en la zona litoral "Áreas Importantes para la Conservación de las Aves" (AICAs), como la Isla Doña Sebastiana, la Península de Chocoy, cerro Amortajado y el humedal de Lenqui. Cabe destacar que los humedales costeros ubicados en la comuna de Maullín forman parte de le Red Hemisférica de Reservas para Aves Playeras (RHRAP) (Andres et al., 2009). Se añade, por último, la imponente y extensa playa Mar Brava y la nutrida biodiversidad marina que alberga la punta Chocoy y la Isla Doña Sebastiana, sitios de importancia para la reproducción de aves y mamíferos marinos.

$\mathrm{Al}$ respecto, un dirigente señala:

"tenemos humedales cerca para avistamiento de aves que llegan de todas partes del mundo, tenemos un humedal que está en Lenqui que está integrado al ECMPO, y tenemos otro que no está integrado pero que está muy cerca en Peime"18.

Existe actualmente preocupación mundial por el cuidado de los humedales, los cuales son esenciales para la biodiversidad, albergando más de cien mil especies conocidas de agua dulce (Convención sobre los humedales, Ramsar, 2020) ${ }^{19}$. El dirigente de una de las comunidades indígenas destaca: "lo que buscamos es proteger, no trabajar lo que está en veda, las especies fuera de medida, la idea es regularlo todo para que después tengamos" ${ }^{20}$.

A pesar del reconocimiento de la importancia de la biodiversidad en el bienestar humano, Roberts et al. (2015) señalan que la cuestión de cómo clasificar y/o comprender los aspectos del bienestar asociados a los servicios ecosistémicos que brinda la biodiversidad no se han estudiado en la misma escala. Producto, principalmente, de la primacía de la evaluación económica y ecológica de los servicios, en detrimento de perspectivas sociales o antropológicas que releven las percepciones y conocimientos locales de la biodiversidad. De esta manera, reconocemos que una política pública que otorga a comunidades indígenas locales la administración de este espacio marino-costero, sobre la base de sus usos y conocimientos del ecosistema, contribuye al cuidado, inclusión y sostenibilidad de este territorio (Araos et al., 2020a).

Vulnerabilidad ambiental: Debido a la riqueza natural del territorio, Carelmapu continuamente se ve amenazado por propuestas de instalación de grandes emprendimientos industriales, particularmente, de la acuicultura y la producción de energía. Respecto a lo anterior, una de las dirigentes declara: "nosotros si no hubiésemos in-

18. Entrevista realizada el 11 de Octubre de 2019. Carelmapu, Comuna de Maullín.

19. Consultado en www.ramsar.org el 01 Marzo de 2020.

20. Entrevista realizada el 16 de Enero de 2020. Carelmapu, Comuna de Maullín. 
gresado ese ECMPO no hubiésemos tenido como saber que había una Telefónica del Sur, que había una salmonera y que venía un parque eólico" ${ }^{21}$.

Ante la acelerada degradación del medio ambiente de esta zona del país (Anbleyth-Evans et al., 2020), se vuelve fundamental otorgar un marco de protección jurídica que permita la regulación y protección de la biodiversidad existente en el maritorio y costa de Carelmapu.

\section{Contribuciones sociales}

Participación comunitaria: La participación comunitaria se alza como una forma de acción colectiva para la solución de problemas, con el objetivo de promover el cambio social en el entorno local (Contreras, 200o). Siguiendo a Fernández, Nina y Vargas (2018), la participación de actores locales y sus comunidades tiene un significativo alcance para el desarrollo, permitiendo plantear soluciones a problemáticas desde una perspectiva local y abriendo espacios de planificación en conjunto a agentes de desarrollo. El ECMPO Carelmapu abarca la participación de seis comunidades indígenas locales, 150 personas aproximadamente, las cuales deben tomar en conjunto las decisiones respecto al avance de la solicitud. La importancia de la participación comunitaria dentro del desarrollo local, radica en el valor del compromiso que tienen las comunidades con el territorio que habitan, transformándolos en verdaderos protectores ambientales (Appiah-Opoku, 2007).

Es así, como dirigentes manifiestan que la participación de la comunidad es indispensable, ya que son todos parte del territorio, declarando que: “yo creo que ahí está el desarrollo humano, cuando somos capaces de tomar decisiones, somos capaces de proponer, de ponernos de acuerdo y proyectarnos, buscar solucionar nuestros propios problemas" 22 .

Se visualiza, así, un aporte de carácter comunitario que impulsa la toma de decisiones en conjunto, abordando la solución de problemáticas locales de forma participativa.

Educación ambiental: En Carelmapu los miembros de las comunidades indígenas que desarrollan laborales ligadas al mar y sus costas declaran que: "los recursos se están perdiendo y a través del ECMPO sería más como un registro de los mariscos que se están sacando, porque se está sacando mucho la semilla”23.

21. Entrevista realizada el 19 de Enero de 2020. Carelmapu, Comuna de Maullín.

22. Entrevista realizada el 11 de Octubre de 2019. Carelmapu, Comuna de Maullín.

23. Entrevista realizada el 22 de Enero de 2020. Puerto Montt. 
Esto da cuenta de malas prácticas de extracción de los recursos del mar, las cuales repercuten en su reproducción y sostenibilidad en el tiempo. A partir de esta constatación, la educación ambiental se plantea como una herramienta para proyectar la sostenibilidad de los recursos naturales existentes en el territorio, donde los educadores puedan contribuir a que la población reciba una nueva percepción de los problemas y desafíos ambientales locales (Sierra, Bustamante y Morales, 2016).

Desarrollo local: El otorgamiento de la administración de un espacio marino-costero a las comunidades locales, en este caso a los pueblos indígenas, tiene el potencial para promover un desarrollo anclado en el territorio y su gente: "acá tenemos la posibilidad de hacer las cosas distintas, de que nos pongamos de acuerdo y seamos nosotros quienes decidamos como queremos vivir" ${ }^{24}$. Se refiere, por tanto, a una opción de desarrollo que busca mejorar la calidad de vida de las personas a partir de las dinámicas socio-productivas que se originan y anclan en el espacio local (Artigas, Ramos y Vargas, 2014).

Empoderamiento: El empoderamiento se entiende como un proceso en el cual se toma conciencia de la exclusión, ya sea esta de carácter social, política, económica o cultural, dando paso a la construcción de un poder que permite favorecer la capacidad de acción colectiva en torno a intereses comunes de los sectores marginados (Contreras, 200o). De esa manera, observamos que el ECMPO Carelmapu ha entregado la oportunidad para que la población, especialmente los y las dirigente/as locales, puedan participar en los procesos de toma de decisión locales y regionales. Una dirigenta plantea al respecto: "el hecho de ya poder detener a las empresas, ya hemos protegido una gran parte" ${ }^{25}$. Siguiendo a Fernández et al. (2018) el empoderamiento local guarda estrecha relación con el nivel de importancia de desarrollo territorial, pues su éxito radicará en la sostenibilidad de la participación de actores locales y en la diferenciación que estos le puedan otorgar. De acuerdo a lo anterior, otro dirigente señala: "antes de proponer grandes cosas, tenemos que decir, esto es nuestro" ${ }_{26}$.

Distribución de poder: Observamos que la creación tanto del ECMPO Carelmapu, así como de otros casos en la Región de Los Lagos, está incentivando un reordenamiento de las fuerzas políticas sobre la base de la recuperación de los bienes comunes del mar por parte de comunidades indígenas y sus alianzas con otros actores relevantes (Diestre y Araos, 2020). Sobre la base de esa redistribución del poder se plantea una idea de gobernanza ambiental sostenida por la participación local (Araos y Ther, 2017), donde los usuarios directos de los recursos naturales pertenecientes o no la cultura mapuche, serían los actores directos de la toma de decisiones respecto al uso y extracción de los recursos del mar. Al respecto un dirigente señala:

24. Entrevista realizada el 11 de Octubre de 2019. Carelmapu, Comuna de Maullín.

25. Entrevista realizada el 22 de Enero de 2020. Puerto Montt.

26. Entrevista realizada el 11 de Octubre de 2019. Carelmapu, Comuna de Maullín. 
"queremos hacer charlas y reuniones y preguntarle al resto de la gente si está de acuerdo, no de tomarnos atribuciones nosotros mismos, primero consultar y después actuar, al final el ECMPO es para todos, para toda la gente que trabaja en el mar, siendo mapuche y no mapuche" ${ }^{27}$.

Calidad de vida: Si bien no existe una definición única del concepto de calidad de vida debido a su ambigüedad y similitud con otros conceptos, es posible definir la calidad como una expresión que nace a raíz de los daños provocados por el proceso de industrialización y el modelo de desarrollo que ha deteriorado gravemente el medioambiente, el cual se aproxima más a la satisfacción de las necesidades humanas básicas, antes que a las exigencias de la economía (De Galindo \& De Llergo, 2017). De esta manera, para uno de los dirigentes: "la calidad de vida depende de cómo cuides tu casa, en este caso de cómo se cuida el territorio" ${ }^{28}$.

Esta declaración expone la importancia que para los pueblos indígenas de Carelmapu tiene la protección de la zona costera, entendiéndola como una tela de relaciones entre los seres humanos y el medio ambiente que se inscribe en el territorio.

\section{Contribuciones económicas}

Sostenibilidad de los recursos naturales: Un dirigente de las comunidades indígenas de Carelmapu plantea: "la idea de nosotros es proteger las especies y que haya abundancias después, que no se acabe" ${ }^{29}$. Observamos que el mantenimiento de los recursos marinos de Carelmapu es fundamental para el modo de vida de las comunidades indígenas, por lo que cualquier alteración en el medioambiente mermará la capacidad del ecosistema de proveer servicios que son esenciales para la existencia y reproducción de este grupo humano. Para Salas, Yaguar, Quishpi y Cutiño (2020) "el impacto del cambio climático sobre la salud de las personas se vuelve un tema más complejo cuando se toma en cuenta que la "salud" no es solo la ausencia de enfermedad sino es el bienestar general de la persona y que el "ambiente" que rodea a cada persona es el principal condicionante de ese bienestar" (Salas et al., 2020, p. 4). De esta manera, y tras el desafío mundial de revertir los cambios negativos de los ecosistemas (MEA, 2005), proteger y restaurar los sistemas naturales de Carelmapu es un principio clave para el futuro del territorio.

Desarrollo local: la vertiente económica del desarrollo local asociada al ECMPO se relaciona con la posibilidad de sostener nuevos emprendimientos locales que fomenten la autonomía económica, como el turismo de intereses especiales o el cultivo

27. Entrevista realizada el 22 de Enero de 2020. Puerto Montt.

28. Entrevista realizada el 11 de Octubre de 2019. Carelmapu, Comuna de Maullín.

29. Entrevista realizada el 16 de Enero de 2020. Carelmapu, Comuna de Maullín. 
de recursos marinos. Uno de los dirigentes menciona: "apostamos a una serie de emprendimientos, que no sean masivos y que sean sustentables, sostenibles y que sean desarrollados por las propias personas" ${ }^{{ }_{30}}$.

Estas ideas nacen tras el reconocimiento y de la toma de conciencia de las riquezas naturales que posee el territorio y de su potencial económico a través del turismo: "el turismo debería ser una clave de desarrollo fundamental, considerando también de que tenemos otras riquezas, tenemos humedales cerca para avistamiento de aves que vienen de todas partes del mundo" ${ }^{31}$.

Si bien se plantea la necesidad de un desarrollo para la localidad, este se aborda desde una óptica de baja escala, el cual no tenga consecuencias medioambientales para el territorio. Por otro lado, se proyecta la participación de los mismos pobladores en estas iniciativas, aumentando el sentido de pertenencia y evitando la migración de los jóvenes Carelmapinos y Carelmapinas: "volverían nuestros jóvenes a trabajar, a estar con nosotros, porque nunca se protegió" ${ }^{2}$.

\section{Contribuciones culturales}

Kûme Mongen: el aporte cultural del buen vivir se instala como una alternativa viable de desarrollo indígena que corre en paralelo al neoliberalismo, ligado al uso sustentable de los recursos de un territorio y la reproducción de la cultura (Mandel, 2017). Siguiendo a uno de los dirigentes del ECMPO Carelmapu:

"tiene que haber una sensibilidad por parte de todos los actores en torno a cómo cuidamos el medio ambiente y que decisiones tomamos, en ese concepto poner en relieve la cultura mapuche es fundamental, es volver a re levantarla y ponerla encima de la mesa como una alternativa de vida, una opción de desarrollo" ${ }^{33}$.

Si bien la existencia de este paradigma indígena se ancla en la ancestralidad de la cultura mapuche, hoy en día adquiere un renovado sentido, al posicionarse como una alternativa ética que puede hacer frente a la crisis ambiental que vive el planeta (Araos et al., 2019). Al respecto, se señala que:

30. Entrevista realizada el 11 de Octubre de 2019. Carelmapu, Comuna de Maullín.

31. Entrevista realizada el 11 de Octubre de 2019. Carelmapu, Comuna de Maullín.

32. Entrevista realizada el 19 de Enero de 2020. Carelmapu, Comuna de Maullín.

33. Entrevista realizada el 11 de Octubre de 2019. Carelmapu, Comuna de Maullín. 
"el Kume Mongen tiene que ver con el vivir bien, y el vivir bien tiene una relación con la tranquilidad espiritual y el equilibrio espiritual del ser humano (...) el ECMPO busca poner en relieve la cosmovisión mapuche, esta visión de que el territorio es uno solo, que en este territorio somos parte de una cadena, y si queremos seguir existiendo tenemos que cuidarlo"34.

Las comunidades que integran este paradigma a sus relatos declaran que: "se acabaría el buen vivir de un territorio dependiendo de una empresa, porque independientemente de cómo uno lo mire es negativo" ${ }^{35}$. Asumiendo en este proyecto la responsabilidad del mantenimiento de su actual modo de vida, sin intervenciones industriales en su espacio marino y costero.

Un comunero de Carelmapu declara en referencia a los emprendimientos industriales:

"ellos hablan de desarrollo, y para mí no hay desarrollo cuando una empresa de esa magnitud llega a un territorio y solamente llega a destruir, y los habitantes, los lamuenes, ¿qué es lo que tienen que hacer? Irse porque acá los lamuenes viven del mar" ${ }^{36}$.

Es importante señalar que los conocimientos de las comunidades indígenas entrevistadas a acerca de las cosmovisiones y prácticas asociadas al Kume Mongen no se manifiestan en todas las comunidades por igual, declarando dos de las seis desconocer esta visión del buen vivir. Esta situación se explicaría por la reciente formación de estas dos comunidades que integran el ECMPO Carelmapu, las cuales manifiestan ser las últimas en el territorio en haberse constituido.

A pesar de lo anterior, se observa también un creciente interés por parte de los dirigentes en el rescate del patrimonio indígena y el fomento de prácticas culturales en el territorio, como medicina tradicional mapuche basada en el uso de plantas y hierbas, talleres de lengua, entre otros.

Si bien vemos que las comunidades indígenas en ocasiones carecen de los conocimientos teóricos para reconocer el alcance de los saberes asociado al buen vivir, estas guardan un conocimiento práctico y empírico que es fundamental para la mantención de sus modos de vida. De acuerdo a lo anterior y refiriéndose a los saberes mapuche, una dirigenta declara: "uno cree que va sin ningún conocimiento, pero en realidad uno tiene mucho conocimiento porque te criaste como mapuche, porque antiguamente no se hablaba de ser mapuche en la familia, pero se vivía y se hacían cosas como mapuche" ${ }^{37}$.

34. Entrevista realizada el 11 de Octubre de 2019. Carelmapu, Comuna de Maullín.

35. Entrevista realizada el 22 de Enero de 2020. Carelmapu, Comuna de Maullín.

36. Entrevista realizada el 22 de Enero de 2020. Carelmapu, Comuna de Maullín.

37. Entrevista realizada el 19 de Enero de 2020. Carelmapu, Comuna de Maullín. 
Prácticas culturales: La reproducción de la cultura bajo el desarrollo de los usos consuetudinarios es uno de los desafíos colocados por los ECMPO en los diversos territorios (Mandel, 2017). Las contribuciones y beneficios culturales que se pueden visualizar son, por un parte, la integración de la cosmovisión mapuche respecto al cuidado del entorno, poniendo de relieve su forma de vida como una opción de desarrollo, y por otro lado, la activación de la memoria e identidad de las comunidades indígenas locales. $\mathrm{Al}$ respecto una de las dirigentes de las comunidades indígenas señala: "decidimos formar una comunidad para trabajar todo lo que es el tema cultural, para volver a reconocernos como mapuche, ese fue nuestro primer foco" ${ }^{138}$.

De esta manera, "encontramos en la demanda de las comunidades indígenas, la búsqueda de derechos sobre el territorio para poder ejercer las prácticas culturales y económicas que dan sustento a las familias y continuidad a la Identidad Lafkenche" (Mandel, 2017, p. 25).

Identidad territorial: La identidad territorial se define como la cercanía y pertenencia de las comunidades indígenas con ciertos territorios, los cuales poseen una amplia significación cultural o en los que estas se desarrollan económicamente (Mandel, 2017; Marileo, 2002). En relación a lo anterior se posiciona la identidad Lafkenche o gente del mar como un espacio de construcción identitaria en potencia. Una dirigenta declara con mucha entereza: "nosotros con la Ley Lafkenche tenemos como defendernos" ${ }^{\prime 3}$.

Libre determinación: Se puede definir la libre determinación como la libertad de los pueblos indígenas de la toma de decisiones respecto a los asuntos que afectan su vida y su futuro (Fernández y Salinas, 2012). Al respecto un dirigente de una comunidad indígena declara que: "somos nosotros quienes deberíamos tomar el rumbo, diseñar el futuro del espacio que queremos vivir y como queremos vivirlo" 40 .

Respecto a lo planteado, la Ley Lafkenche y la creación de los ECMPOs se visualizan como un importante aporte en materia de apertura de espacios para el ejercicio de la libre determinación de las comunidades indígenas costeras, siendo esta política una posibilidad de delegación explícita de autoridad sobre su territorio (Seelau y Seelau, 2012).

38. Entrevista realizada el 22 de Enero de 2020. Carelmapu, Comuna de Maullín.

39. Entrevista realizada el 22 de Enero de 2020. Carelmapu, Comuna de Maullín.

40. Entrevista realizada el 11 de Octubre de 2019. Carelmapu, Comuna de Maullín. 


\section{Discusión}

Los resultados presentados confluyen en la idea de la necesidad de una protección legal del territorio, lo que otorgaría un conjunto de beneficios ambientales, sociales, económicos y culturales. La protección de la biodiversidad se presenta como un componente esencial para el desarrollo local, que al verse afectada influiría en su capacidad para generar distintos servicios ecosistémicos esenciales para el bienestar humano de los habitantes de la localidad de Carelmapu. Concordamos de esta manera con Roberts et al. (2015), quienes en un estudio sobre Nueva Zelanda señalan que los servicios ecosistémicos que entrega la biodiversidad contribuyen al bienestar de los habitantes: proveyendo alimentos para la subsistencia, sosteniendo la biodiversidad, incremento de la resiliencia y seguridad socio-ecológica de los territorios, y en consecuencia, contribuyendo en el mantenimiento de los modos de vida de los pueblos.

El desgaste y sobre-explotación de algunos recursos marinos se ha posicionado en el territorio como una controversia socio-ambiental donde se enfrentan distintas cosmovisiones e intereses. Por un lado, se encuentran los pescadores artesanales quienes, a través de las Áreas de Manejo y Explotación de los Recursos Bentónicos (AMERB), acceden y explotan los recursos bentónicos con foco al manejo y utilización económica de los bienes comunes. Mientras que, por otro lado, se identifican los intereses de los pueblos indígenas quienes visualizan el ECMPO como una herramienta de administración del maritorio con objetivos de conservación y explotación sustentable de los recursos que reoriente el desarrollo local hacia el resguardo de modos de vida locales (Ebel, 2019).

Se observa, por lo tanto, una controversia local asociada a la sobreposición de diferentes arreglos institucionales para normar el uso y explotación de recursos comunes (Diestre y Araos, 2020), cuya resolución requiere de alianzas y estrategias de colaboración que aún no logran materializarse. Así, la potencialidad del ECMPO como una herramienta de desarrollo local requiere para su despliegue territorial de la participación de todos los interesados o stakeholders, un imperativo que, como observamos en el caso estudiado, está en desarrollo y en vías de alcanzarse.

Cuando analizamos el conjunto de contribuciones de los ECMPOs al bienestar humano se revela que éstos entregan a los habitantes de las localidades costeras una herramienta institucional para controlar potenciales intervenciones en el territorio, que puedan afectar la integridad de los ecosistemas y, consecuentemente, la provisión de servicios ambientales que permitan sostener sus modos de vida (Anbleyth-Evans et al., 2020; Araos et al., 2020a).

Es así, como surge desde una mirada de redistribución del poder un tipo de gobernanza ambiental de base local (principalmente a nivel municipal) anclada en un arreglo institucional de conservación ambiental, donde los actores locales son capaces de participar en los procesos de toma de decisiones que afectan su bienestar. Un proceso 
de aprendizaje que, además, puede motivar el desarrollo de nuevos proyectos locales en otras áreas o dimensiones (Fernández et al., 2018).

La solicitud del ECMPO de Carelmapu, al integrar seis comunidades indígenas locales, no solo propone una nueva forma de gobernanza ambiental, sino que también una nueva forma de distribución del poder que camina hacia la libre determinación los pueblos indígenas. Si bien nuestro país se adscribe al reconocimiento legal de este derecho, por medio del Convenio 169 de Organización Internacional del Trabajo (OIT), éstos no se practican o son recurrentemente negligenciados (Seelau y Seelau, 2012). Por lo tanto, con la implementación del ECMPO se abren posibilidades para el ejercicio de la soberanía territorial.

Con relación a los indicadores propuestos, destacamos algunos que ya han sido registrados en este estudio; como el empoderamiento, la distribución de poder y la participación comunitaria de las comunidades indígenas, lo que ha permitido integrar a este grupo social en el proceso de toma de decisiones que involucran las problemáticas marino-costeras de la localidad.

En el ámbito cultural se distinguen indicadores como la manifestación de prácticas culturales asociadas a los modos de vida litorales, así como el fortalecimiento de la identidad cultural indígena, gracias al desarrollo de iniciativas de medicina tradicional, lengua y tradiciones culturales.

Respecto de otros indicadores ambientales, sociales, económicos y culturales no observados en el presente estudio, se reconoce que dependen para su despliegue y expresión en el territorio del decreto de creación del ECMPO y la posterior elaboración del plan de administración.

En suma, el presente trabajo entrega elementos para visibilizar los potenciales indicadores que permitirían evaluar y medir la eficacia de esta política pública, considerando su contribución al bienestar humano de las comunidades indígenas que solicitan y administran un ECMPO. Su importancia finalmente radica en la potencialidad que estos indicadores puedan apoyar la implementación de la ley Lafkenche sobre la base de evidencia medible, observable y verificable.

\section{Conclusiones}

El establecimiento de un ECMPO tiene gran incidencia en el bienestar humano de la comunidad que lo acoge. Se identifican amplias contribuciones en materia ambiental, social, económica y cultural, las cuales permitirían mejorar la calidad de vida de las comunidades asociadas a una solicitud. Entre éstas, se destaca: la protección de la biodiversidad y sus efectos sobre la sostenibilidad ambiental del territorio/maritorio; la participación comunitaria y sus consecuencias en la redistribución del poder a nivel local/regional; y la libre determinación, como un ejercicio de soberanía territorial de los pueblos indígenas a través de medios institucionales. Actualmente, el bienestar 
humano de la localidad de Carelmapu se ve amenazado por la sobreexplotación de recursos del mar y la instalación de grandes emprendimientos industriales acuícolas, cuyos impactos pueden afectar la provisión de los servicios ambientales. Es innegable, por lo tanto, la relevancia de una medida de protección del maritorio que sea apropiada, legítima e inclusiva.

Por otro lado, la ley Lafkenche abre una relación entre comunidades indígenas y el Estado que se visualizaba debilitada, otorgando un espacio para el reconocimiento de los derechos indígenas. Se fortalecen de esta manera las identidades territoriales, las cuales van tomando una connotación política en el marco de la lucha territorial del pueblo mapuche. Si bien se reconoce a la ley Lafkenche como un gran avance en materia de reconocimiento de derechos territoriales, a lo largo de su aplicación desde el año 2008 a la fecha ésta ha tenido un sin número de problemas para su correcta aplicación. La extensión temporal del proceso de tramitación, la falta de información en torno a los criterios para aprobar o rechazar las solicitudes y la excesiva burocracia, obstaculizan la aplicación de la ley.

Finalmente, la perspectiva del bienestar humano explorada en este artículo permitió analizar una política pública para los pueblos indígenas, reconocer sus beneficios y avanzar en medidas para su evaluación. De esta manera, vemos a los indicadores de bienestar humano de los ECMPOS como una contribución para el análisis de la aplicación de la Ley Lafkenche a nivel nacional, los cuales pueden ser un primer paso para el desarrollo de una estrategia de seguimiento, monitoreo y evaluación en el mediano y largo plazo.

\section{Referencias}

Aguado, Mateo, Diana Calvo, Candela Dessal, Jorge Riechman, José González y Carlos Montes (2012). La necesidad de repensar el bienestar humano en un mundo cambiante. Papeles, (119): 49-76.

Aguado, Mateo (2016). Vivir bien en un planeta finito: Una mirada socio-ecológica al concepto de bienestar humano. (Tesis doctoral) Universidad Autónoma de Madrid.

Andres, Brad, James Johnson, Jorge Valenzuela, Morrison Guy, Luis Espinosa \& Ross Ken (2009). Estimating Eastern Pacific Coast Populations of Whimbrels and Hudsonian Godwits, with an Emphasis on Chiloé Island, Chile. Waterbirds, 32(2): 216224.

Araos, Francisco, Jeremy Anbleyth-Evans, Wladimir Riquelme, Carlos Hidalgo, Francisco Brañas, Emilia Catalán, David Nuñez y Florencia Diestre (2020a). Marine Indigenous Areas: Conservation Assemblages for Sustainability in Southern Chile. Coastal Management, 48 (4): 289-307. Doi: https://doi.org/10.1080/o89207 53.2020.1773212. 
Araos, Francisco, Emilia Catalán, Ricardo Alvarez, David Nuñez, Francisco Brañas y Wladimir Riquelme (202ob). Espacios Costeros Marinos Para Pueblos Originarios: usos consuetudinarios y conservación marina. Revista Anuário Antropológico I (1): 47-68. Doi: https://doi.org/10.400o/aa.4933.

Araos, Francisco, Wladimir Riquelme, Juan Skewes, Ana Vianna, Ricardo Alvarez, Francisco Ther, Allan Iwama, Sarita Albagli, Aderval Costa \& Emmanuel Duarte (2019). La vida después de la devastación: lo común de la tragedia en territorios sociobiodiversos de Chile y Brasil. Antropologías Del Sur, 6(12): 87-106. Doi: https://doi.org/10.25074/rantros.v6i12.1120.

Araos, Francisco \& Francisco Ther (2017). How to adopt an inclusive development perspective for marine conservation: Preliminary insights from Chile. Current Opinion in Environmental Sustainability, 24:68-72. Doi: https://dx.doi.org/10.1016/j. cosust.2017.02.008.

Anbleyth-Evans, Jeremy, Francisco Araos, Francisco Ther-Ríos, Ricardo Segovia \& Vreni Haussermann (2020). Toward Marine Democracy in Chile: examining aquaculture ecological impacts through common property local ecological knowledge. Marine Policy,113, 10369o. Doi: https://doi.org/10.1016/j.marpol.2019.10369o.

Appiah-Opoku, Seth (2007). Indigenous beliefs and environmental stewardship: a rural Ghana experience. Journal of Cultural Geography, 24(2): 79-98. Doi: https:// doi.org/10.1080/08873630709478212.

Artigas, Elaine, Alejandro Ramos \& Heriberto Vargas (2014). La participación comunitaria en la conservación del medioambiente: clave para el desarrollo local sostenible. Revista DELOS: Desarrollo Local Sostenible, 21. Recuperado de http:// www.eumed.net/rev/delos/21/conservacion.html.

Asesoría técnica Parlamentaria (2018). La ley Lafkenche, análisis y perspectivas a 10 años de su entrada en vigor. Recuperado de www.bibiliotecanacionaldigital.gob.cl.

Canales, Manuel (2006). Metodologías de la investigación social. Santiago: LOM ediciones.

Contreras, Rodrigo (200o). Empoderamiento Campesino y Desarrollo Local. Revista Austral de Ciencias Sociales, 4, 55-68. Doi: https://doi.org/10.4206/rev.austral. cienc.soc.20oo.n4-03.

Costanza, Robert, Brendan Fisher, Saleem Ali, Caroline Beer, Lynne Bond, Roelof Boumans, ... \& Dianne Gayer (2007). Quality of life: An approach integrating opportunities, human needs, and subjective well-being. Ecological economics, 61(23): 267-276. Doi: https://doi.org/10.1016/j.ecolecon.2006.02.023. 
Costanza, Robert, Lew Daly, Lorenzo Fioramonti, Enrico Giovannini, Ida Kubiszewski, Lars Mortensen, Kate Pickett, Kristin Vala, Roberto De Vogli \& Richard Wilkindon (2019). Modelling and measuring sustainable wellbeing in connection with the UN Sustainable Development Goals. Ecological Economics, 130, 350-355. Doi: http://dx.doi.org/10.1016/j.ecolecon.2016.07.009.

Cursach, Jaime (2018). Revisión bibliográfica sobre la biodiversidad marina del mar adyacente a Carelmapu, con especial énfasis en aves y mamíferos marinos. Documento inédito.

Cruz de Galindo, Luz \& Ana López de Llergo (2017). Desarrollo comunitario y calidad de vida. Revista Panamericana de Pedagogía. Saberes y quehaceres del pedagogo, 4:57-56. Recuperado a partir de https://revistas.up.edu.mx/RPP/article/view/1883.

Delgado, Luisa, Pamela Bachmann \& Bárbara Oñate (2007). Gobernanza ambiental: una estrategia orientada al desarrollo sustentable local a través de la participación ciudadana. Ambiente y Desarrollo, 23(3): 68-73.

Diestre, Florencia \& Francisco Araos (2020). La recuperación de los comunes en el sur-austral: construcción institucional de Espacios Costeros Marinos de Pueblos Originarios. Polis, 19(57): 19-50.

Ebel, Sarah (2019). In Hot Water: A Multi-Level Analysis of Structure, Agency, and Adaptive Governance in Chile's Lake Region. Electronic Theses and Dissertations, 3097. Recuperado de https://digitalcommons.library.umaine.edu/etd/3097.

Fernández, Gabith, Dante Ayaviri \& Ruth Maldonado (2018). Participación de los actores en el desarrollo local en entornos rurales. Revista de ciencias sociales, 24(3): 62-82. Doi: https://doi.org/10.31876/rcs.v24i3.24922.

Fernández, Margarita \& Javier Salinas (2012). Defensa de los derechos territoriales en Latinoamérica. RIL editores, Santiago, Chile.

Gaona Pando, Georgina (2013). El derecho a la tierra y protección del medio ambiente por los pueblos indígenas. Nueva antropología, 26(78): 141-16. Recuperado de http://www.scielo.org.mx/scielo.php?script=sci_arttext\&pid=So18506362013000 $100007 \& \operatorname{lng}=$ es\&tlng=es.

Gissi, Nicolás, Daniela Ibacache, Bernardo Pardo y Cristina Nancucheo (2017). El Estado chileno, los lafkenche y la Ley 20.249: ¿Indigenismo o política del reconocimiento? Revista Austral de Ciencias Sociales, 32:5-21. Doi: https://doi.org/10.4206/ rev.austral.cienc.soc.2017.n32-01.

González, Raúl, María Olivares, Jennifer López \& Osmaida Estrada (2020). Sostenibilidad de los ecosistemas y su efecto en el bienestar humano. Revista UNIANDES de Ciencias de la Salud, 3(1): 391-406. 
Hasen, Felipe (2012). La noción de Küme Mogñen en el pueblo mapuche: aproximaciones desde un enfoque ecosistémico. Kula, Antropólogos del Atlántico Sur, 7, 96-114.

Hiriart-Bertrand, Luciano, Juan Silva \& Stefan Gelcich (2020). Challenges and opportunities of implementing the marine and coastal areas for indigenous peoples policy in Chile. Ocean \& Coastal Management, 193:105233. doi: 10.1016/j.ocecoaman.2020.105233.

Instituto Nacional de Estadística (2019). División Política Administrativa y Censal. Departamento de Geografía. Chile.

Kilpatrick, Marm, Daniel Salkeld, Georgia Titcomb \& Micah Hahn (2017). Conservation of biodiversity as a strategy for improving human health and well-being. Philosophical Transactions of the Royal Society B: Biological Sciences, 372(1722): 20160131.

Mandel, Astrid (2017). Las dirigentas Lafkenche: su incidencia en la demanda territorial y política de la Organización Identidad Territorial Lafkenche y la influencia de la organización en su ser mujeres Mapuche (Tesis de postgrado). Facultad de Ciencias Sociales, Universidad de Chile. Chile.

Marileo, Armando (2002). Mundo mapuche. Meridión Comunicaciones, Chile.

Martín-López, Berta, José González, Sandra Díaz, Isabel Castro y Marina GarcíaLlorente (2007). Biodiversidad y bienestar humano: el papel de la diversidad funcional. Ecosistemas, 16(3): 69-80.

Millennium Ecosystem Assessment (2005). Ecosystems and human well-being: synthesis. Island, Washington, DC.

Nahuelpan, Héctor (2016). Micropolíticas mapuche contra el despojo en el Chile neoliberal. La disputa por el lafkenmapu (territorio costero) en Mehuín. Izquierdas, 30:89-123. Doi: http://dx.doi.org/10.4067/So718-50492016000500004.

Pascual, Unai, Patricia Balvanera, Sandra Díaz, Gyorgi Pataki, Eva Roth, Marie Stenseke,... \& Noboyuki Yagi (2017). Valuing nature's contributions to people: the IPBES approach. Current Opinion in Environmental Sustainability, 26, 7-16.

Raworth, Kate, Caroline Sweetman, Swati Narayan, Jo Rowlands \& Adrienne Hopkins (2012). Conducting semi-structured Interviews. Oxfam.

Roberts, Lin, Ann Brower, Geoff Kerr, Simon Lambert, Wendy McWilliam, Kevin Moore, John Quinn, David Simmons, Simon Thrush, Mike Townsend, Paul Blaschke, Robert Costanza, Ross Cullen, Ken Hughey y Steve Wratten (2015). The nature of wellbeing: how nature's ecosystem services contribute to the wellbeing of New Zealand and New Zealanders. Department of Conservation, Wellington. 
Rodríguez, Daniel, Claudio Gajardo \& Francisco Ther (2014). Carelmapu, Provincia de Llanquihue, Región de Los Lagos. Serie etnografías Litorales. Chile litoral 2025: Modelo de Gestión Territorial para Asentamientos de Pescadores Artesanales. Universidad de Los Lagos, Osorno.

Rojas, Nicolás \& David Soto (2016). Küme Mongen: el buen convivir mapuche como alternativa de desarrollo humano y sustentable. Ponencia III Congreso social: Ecología humana para un desarrollo sostenible e integral. 4-10-2016, Universidad Católica de Chile, Santiago.

Seelau, Laura \& Ryan Seelau (2012). Entre todo y nada: la libre determinación de los pueblos indígenas y los espacios para su ejercicio en Chile. En: M. Fernández \& G. Salinas, ed., Defensa de los derechos territoriales en Latinoamérica, 1st ed. [online] Santiago, Chile: RIL editores, pp.29-69. Recuperado de: https://books.google.es/ books?id=QKfAt3e $7 \mathrm{r} 88 \mathrm{C} \& \mathrm{lpg}=\mathrm{PP} 1 \& \mathrm{hl}=\mathrm{es} \& \mathrm{pg}=\mathrm{PR} 1 \# \mathrm{v}=$ onepage $\& \mathrm{q} \& \mathrm{f}=$ false .

Severiche-Sierra, Carlos, Edna Gómez-Bustamante \& José Jaimes-Morales (2016). La educación ambiental como base cultural y estrategia para el desarrollo sostenible. Telos: Revista de Estudios Interdisciplinarios en Ciencias Sociales, 18(2): 266-281.

\section{Sobre los autores}

Daniça Cid es Trabajadora Social y Licenciada en Trabajo Social de la Universidad de Los Lagos. Sus intereses de investigación son las dimensiones sociales de la conservación de la biodiversidad y el área socio-comunitaria en general. Correo Electrónico: danica.cid.gajardo@gmail.com. https://orcid.org/0000-0002-5253-1044.

Francisco Araos es Antropólogo Social, Doctor en Ambiente y Sociedad. Profesor Asociado de la Universidad de Los Lagos, Investigador del Centro de Estudios del Desarrollo Regional y de Políticas Públicas, Osorno. Sus intereses de investigación son las dimensiones humanas de la conservación de la biodiversidad, el manejo de recursos naturales, los pueblos indígenas y locales, y el cambio ambiental global. Correo Electrónico: francisco.araos@ulagos.cl. (iD https://orcid.org/0000-0002-7713-8230 


\title{
CUHSO
}

Fundada en 1984, la revista CUHSO es una de las publicaciones periódicas más antiguas en ciencias sociales y humanidades del sur de Chile. Con una periodicidad semestral, recibe todo el año trabajos inéditos de las distintas disciplinas de las ciencias sociales y las humanidades especializadas en el estudio y comprensión de la diversidad sociocultural, especialmente de las sociedades latinoamericanas y sus tensiones producto de la herencia colonial, la modernidad y la globalización. En este sentido, la revista valora tanto el rigor como la pluralidad teórica, epistemológica y metodológica de los trabajos.

\author{
EDITOR \\ Matthias Gloël \\ CoOrdinadora EDITORIAL \\ Claudia Campos Letelier \\ Corrector de ESTILO Y DiSEÑAdor \\ Ediciones Silsag \\ Traductor, CORRECTOR LENGUA INGLESA \\ Aurora Sambolin Santiago \\ SITIO WEB \\ cuhso.uct.cl \\ E-MAIL \\ cuhso@uct.cl
}

LICENCIA DE ESTE ARTÍCULO

Creative Commons Atribución Compartir Igual 4.0 Internacional 